\title{
EREBEA
}

Revista de Humanidades

y Ciencias Sociales

NúM. 1 (2011), pp. 137-156

ISSN: 0214-0691

\section{La serpe in corpo. Per il bestiario di Giordano DA Pisa}

Nicolo Maldina

Universidad de Pisa

RESUMEN

A fronte del riconoscimento, nelle artes praedicandi, dell'efficacia retorica delle immagini tratte dal mondo animale pare opportuno rileggere i numerosi animali presenti nei sermoni tardo-medievali avvertiti del retroterra culturale dell'imagery zoologica medievale. Nell'articolo si propongono alcune osservazioni in questo senso a partire dall'analisi di una similitudine di Giordano da Pisa, in cui ai demoni che vengono cacciati astenendosi dai diletti corporali viene associato il digiuno come unica risorsa per l'uomo nel cui stomaco si è introdotto un serpente per vivervi da parassita.

Palabras Clave

Bestiario, Giordano da Pisa, Exemplum, Agiografia, Digione.

Fecha de recepción: 8 de febrero de 2011

Fecha de aceptación: 1 de marzo de 2011

\section{Abstract}

In view of the recognition, in the artes praedicandi, of the rhetorical effectiveness of images taken from the animal world, it seems opportune to review the many animals found in late medieval sermons warned of the cultural background of medieval zoological imagery. The article offers some observations in this respect, starting from the analysis of a simile by Giordano da Pisa, in which demons expelled abstaining from bodily pleasures are associated with fasting as a resource for the man in whose stomach has introduced a snake to live there as a parasite.

\section{KeYwORDS}

Bestiary, Giordan da Pisa, Exemplum, Agiography, Fasting. 

O tortuose serpens, qui mille per meandros

frondesque flexuosas agitas quieta corda, disce, Christus hic est. Hic Christus est, liquesce! (Prudenzio, Cathemerinon, vv. 141-46)

Nel secondo libro del De doctrina christiana, quando il discorso volge a trattare dell'ambiguità di quei signa biblici che assumono «ipsae res quas propriis verbis significamus» non nel loro senso proprio, ma «ad aliquid alius significandum», Agostino raccomanda a chi voglia coglierne pienamente il senso di guadagnare una nutrita enciclopedia culturale, così da poter far leva su di un vasto complesso di competenze naturalistiche, astronomiche e numerologiche per decifrare la ricca allusività di simili translata e opporre a una rischiosa ignoranza la ferma sicurezza circa le proprietà degli enti richiamati per via d'analogia nella Bibbia. Si tratta di una materia talmente vasta da spingere Agostino ad auspicare la compilazione di manuali che, sul modello del Chronicon di Eusebio di Cesarea, raccolgano e ordinino «quoscumque terrarum locos quaeve animalia vel herbas atque arbores sive lapides vel metalla incognita speciesque quaslibet scriptura commemorat» e ne passino in rassegna le principali caratteristiche, in modo tale da fornire all'esegeta, riunite in un volume, tutte le claves necessarie alla corretta interpretazione di taluni passi biblici. ${ }^{1}$ Tra queste, in ragione dell'alta frequenza di traslati animaleschi

1 Cfr. De doctrina christiana, II, $\mathrm{x}, 15$ : «Duabus autem causis non intelliguntur quae scripta sunt: si aut ignotis aut ambiguis signis obteguntur. Sunt autem signa vel propria vel translata. Propria dicuntur, cum his rebus significandis adhibetur propter quas sunt instituta, sicut autem dicimus bovem, cum intelligimus pecus quod omnes nobiscum latinae linguae homines hoc nomine vocant. Translata sunt, cum et ipsae res quas propriis verbis significamus, ad aliquid aliud significandum usurpantur, sicut dicimus bovem et per has duas syllabas intelligimus pecus quod isto nomine appellari solet, sed rursus per illud pecus intelligimus evangelistam, quem significavit scriptura interpretante apostolo dicens: "Bovem triturantem non infrenabis"»; xvi, 23: «Rerum autem ignorantia facti obscuras locutiones, cum ignoramus vel animatium vel lapidum vel herbarum naturas aliarumve rerum, quae plerumque in scripturis similitudinibus alicuius gratia ponuntur» e xxxix, 59: «Sicut autem quidam de verbis omnibus et nominibus hebraeis et syris et aegyptiis, vel si qua alia lingua in scripturis sanctis inveniri potest, quae in eis sine interpretatione sunt posita, fecerunt ut ea separatim interpretarentur, [...], quod ergo hi fecerunt de his rebus, ut non sit necesse chri- 
nel linguaggio biblico, un posto non secondario viene riservato alla proprietà degli animali: occorre, infatti, sapere - argomenta Agostino - che il serpente presenta a chi lo vuole colpire tutto il corpo a difesa del capo per comprendere a pieno il senso della raccomandazione evangelica ad essere "prudentes sicut serpentes» come invito per il buon cristiano a difendere Cristo, il capo, con tutto il proprio corpo, così come risulterebbe enigmatico l'imperativo «Intrate per angustam portam» senza sapere che è proprio nelle strettoie delle caverne che il serpente muta la propria pelle e si rinnova alla stessa maniera in cui viene chiesto all'uomo di rinnovare la propria condotta morale, ${ }^{2}$ secondo un assunto non limitato ai

stiano inmultis propter pauca laborare, sic video posse fieri, si quem eorum qui possunt benignam sane operam fraternea utilitati decleret impendere, ut quoscumque terrarum locos quaeve animalia vel herbarum atque arbores sive lapides vel metalla incognita speciesque quaslibet scriptura commemorat, ea generatim dirigens, sola exposita litteris mandet.» (a cura di M. Simonetti, Milano, Mondadori, 2006 3 pp., rispettivamente, 92-94; 110 e 160). Su questi aspetti del pensiero agostiniano cfr., almeno, H.I. Marrou, Saint Augustin et la fin de la culture antique, Paris, De Boccard, 1958, pp. 447-49; N. Henkel, Studien zum Physiologus im Mittelalter, Tübingen, Niemeyer, 1976, p. 140 e F. Zambon, Teologia del bestiario, in Id., L'alfabeto simbolico degli animali. I bestiari del Medioevo, Roma, Carocci, 2001, pp. 23-54, pp. 33-35. La stretta dipendenza tra esegesi biblica e studio degli animali è, d'altronde, un assioma comune alla cultura tardo-antica e costantemente ripetuto lungo tutto il Medioevo: cfr., oltre agli studi già ricordati, almeno F. Sbordone, Ricerche sulle fonti e la composizione del «Physiologus» greco, Napoli, Tip. Torella, 1936; M.I., Gerhardt, Zoologique médiévale: préocupations et procédés, in «Miscellanea medievalia», 7 (1970), pp. 231-48; M.P. Ciccarese, Il simbolismo antropologico degli animali nell'esegesi cristiana antica: criteri e contenuti ermeneutici, in "Annali di storia dell'esegesi», 7/2 (1990), pp. 529-67; Ead., Bibbia, bestie, bestiari: l'interpretazione cristiana degli animali dalle origini al Medioevo, in Il Battistero di Parma. Iconografia, iconologia, fonti letterarie, a cura di G. Schianchi, Milano, Vita e Pensiero, 1999, pp. 375-410; R.M. Grant, Early Christians and animals, London- New York, Routledge, 1999.

2 Cfr. De doctrina christiana, I, xvi, 24: «Nam et de serpente, quod notum est totum corpus eum pro capite obicere ferientibus, quantum inlustrat sensum illum quod Dominus iubet astutos nos esse sicut serpentes, ut scilicet pro capite nostro, quod est Christus, corpus potius persequentibus offeramus, ne fides christiana tamquam necetur in nobis, si parcentes corpori negemus Deum! Vel illud quod per cavernae angustias coartatus, deposita vetere tunica vires novas accipere dicitur, quantum concinit ad imitandam ipsam serpentis astutiam exeundumque veterem hominem, sicut apostolus dicit, ut induamur novo, et exeundum er angustias dicente Domino: "Intrate per angustam portam”!». (ed. cit., p. 110). I riferimenti biblici sono, rispettivamente a Mt. 10, 16 («Estote ergo prudentes sicut serpentes et simplices sicut columbae») e 7, 14 («Intrate per angustam portam, quia lata porta et spatiosa via est, quae ducit ad perditionem, et multi sunt qui intrant per eam. Quam angusta porta et arta via est quae ducit ad vitam, et pauci sunt qui inveniunt eam!»). Si tratta di interpretazioni diffuse della natura dei serpenti biblici, pienamente formulate sin dal Physiologus - si tratta del prima e della quarta natura attribuite dal Fisiologo al serpente nella versione greca del testo - e, come tali, ripetute anche in altre occasioni dallo stesso Agostino (cfr., a titolo esemlificativo, Quaestiones XVI in Matthaeum, 8). Sul simbolismo del serpente nella letteratura cristiana antica cfr., da ultimo, M.P. Ciccarese, Serpente, in Animali simbolici. Alle origini del bestiario cristiano, vol. II (Leone-Zanzara), a cura di Ead., Bologna, Ed. Dehoniane, 2007, pp. 253-83. Si tenga poi conto del fatto che è stato autorevolmente notato come Agostino scelga come esempio il serpente, prevalentemente interpretato in chiave negativa o demonologica sulla base di Gn. 3, 1, per argomentare sulla base dell'ambivalente simbologia dell'animale «quanto la conoscenza dei suoi caratteri e delle 
soli animali, come il serpente, gravati da articolate sovrastrutture simboliche, ma valido nella sostanza per tutti gli animali menzionati nella Bibbia, ciascuno da interpretare scegliendo, tra le diverse proprietà e nature, la più coerente con il senso del contesto della menzione biblica:

Ut ergo notitia naturae serpentis inlustrat multas similitudines quas de hoc animante dare scriptura consuevit, sic ignorantia nonnullorum animalium quae non minus per similitudines commemorat, impedit plurimum intellectum. ${ }^{3}$

Si tratta, com'è noto, di declinazioni del pensiero agostiniano che trovano sicuro riscontro nel contesto dell'esegesi cristiana antica e di posizioni destinate a una lunga fortuna nella cultura medievale. ${ }^{4}$ Tuttavia, piuttosto che insistere sulla stretta relazione tra simili affermazioni e la diffusione di opere che, sul modello del Physiologus greco, indicizzano e interpretano simbolicamente gli animali biblici, ${ }^{5}$ importa qui osservare come sin dalle matrici patristiche della rhetorica Sacra medievale venga riconosciuta ai traslati animaleschi un' essenziale importanza tra le peculiarità dello stile biblico e, più nello specifico, come al fascino di simili traslati zoologici non si riveleranno insensibili quanti, sempre sulla scia del pensiero agostiniano, assumeranno il sermo biblico a modello di una retorica cristianamente intesa e fondata. ${ }^{6}$

sue usanze fosse utile per orientare il senso dell'interpretazione allegorica, là dove la Scrittura ce lo presenta, e interpreta in senso positivo, cristologico ed ecclesiale, cioè quello meno frequente, due usanze del serpente» (M. Simonetti, nota ad. loc., ed. cit., p. 446). Sul principio di ambivalenza simbolica, caratteristico dell'interpretazione simbolica degli animali biblici, si veda L. CharbonneauLassay, Le Bestiaire du Christ, Paris-Liège, Desclèe de Brouwer, 1940, rist. an., Milano, Arché, 1975; H. Henkel, Studien zum Physiologus im Mittelalter cit., pp. 142-43; M.P. Ciccarese, Il simbolismo antropologico degli animali nell'esegesi cristiana antica cit. e F. Zambon, Teologia del bestiario cit.

3 De doctrina christiana, II, xvi, 24 (ed. cit., p. 110).

4 Cfr., in particolare, F. Zambon, Teologia del bestiario cit.

5 Sulla formazione e sulle caratteristiche della tradizione dei bestiari medievali si può ricorrere, accanto ai già citati contributi di Henkel e di Zambon, ad alcuni degli studi più recenti e aggiornati: Beast and Birds of the Middle Ages. The Bestiary and its Legacy, a cura di WW.B. Clark e M.T. McMunn, Philadelphia, University of Pennsylvania Press, 1989; J. Voisenet, Bestiaire chrétienne. L'imagerie animale des auteurs du Haut Moyen Âge ( $V^{e}-X I^{e}$ s.), Toulouse, Presses Universitaires du Mirail, 1994; Id., Bêtes et Hommes dans le monde médiéval. Le bestiaire des clercs du V au XII siécle, Thurnhout, Brepols, 2000; L'animal exemplaire au Moyen Âge (Ve-XV siécle), a cura di J. Berlioz e M.A. Polo de Beaulieu, Rennes, Presses Universitaires de Rennes, 1999.

6 Sulla centralità del pensiero agostiniano, e in particolare del De doctrina christiana, nella formazione della retorica sacra medievale basti il rimando a E. Auerbach, Mimesis. Dargestellte Wirklichkeit in der abendländischen Literatur, Bern, Francke, 1956; Id., Literaturursprache und Publikum in der lateinischen Spätantike und im Mittelalter, Bern, Francke, 1958; H. Caplan, Of Eloquence. Studies in Ancient and Medieval Rhetoric, a cura di A. King e H. North, Ithaca-London, Cornell University Press, 1970 e a J.J. Murphy, Rhetoric in the Middle Ages. A history of Rhetorical Theory 
Non a caso, quindi, tra le cifre stilistiche del sermo modernus che si evincono dalle precise indicazioni messe a punto nelle artes praedicandi a simili risorse retoriche e concettuali viene riservato un posto di assoluto rilievo. ${ }^{7}$ Nell'importante ars di Tommaso di Chobham, ad esempio, tra le competenze "precipue et specialiter omni predicatori necessaria» viene raccomandata proprio una profonda conoscenza delle proprietà e delle nature degli animali:

[...]; tertium est ut sciat naturas animalium et etiam aliarum rerum, quia nihil est quod magis moveat corda auditorum quam proprietates animalium et aliarum rerum de quibus predicatur bene assignate, quia similitudines rerum quasi quedam novitates facilius et delectabilius movent animam. ${ }^{8}$

Alle immagini da bestiario e, più in generale, alle «similitudines rerum» viene, però, assegnato un ruolo di primo piano tra gli strumenti a cui il predicatore può ricorrere per dilatare narrativamente il sermone, oltre che in ragione dell'importante funzione psicagogica qui ricordata - "quia [...] facilius et delectabilius movent animam» -, anche in virtù del generale riconoscimento dell'efficacia argomentativa di simili comparazioni, come lo stesso Tommaso non manca di sottolineare ricordando che "omnis res que persuaderi debet animo uditoris, multo melius insinuabitur per similitudinem quam per simplicem et nudam veritatem,${ }^{9}$ e, in particolare, di fronte al potenziale parenetico di simili traslati: «Nec sufficit eas [scil. proprietates multarum rerum] cognoscere nisi etiam eas ad aedificationem animae sciamus adaptare», argomenta infatti, in un'altra importante ars, Richard di Thetford, ${ }^{10}$ riecheggiando un tópos particolarmente diffuso

from Saint Augustine to the Renaissance, Berkeley, University of California Press, 1974.

7 Dalla vasta bibliografia sull'affermazione del sermo modernus e dell'ars praedicandi ci si limita a rinviare ad alcuni lavori classici: Th.M. Charland, Artes praedicandi. Contribution à l'histoire de la rhétorique au Moyen Âge, Paris-Ottawa, Vrin-Institut d'études médiévales, 1936; C. Delcorno, Giordano da Pisa e l'antica predicazione in volgare, Firenze, Olschki, 1975; M.G. Briscoe, Artes praedicandi/B.H.Jaye, Artes orandi, Turnhout, Brepols, 1992; De l'homélie au sermon. Histoire de la prédication médiévale, actes du Colloque internationale de Louvain-la-Neuve, 9-11 juillet 1992, édités par J. Hamesse et X. Hermand, Louvain-la-Neuve, Institut d'études médiévales, 1993; F. Morenzoni, Des écoles aux paroisses. Thomas de Chobham et la promotion de la prédication au début du XIII siécle, Paris, Institut d'études augustiniennes, 1995; N. Beriou, L'avènement des maîtres de la Parole. La prédication à Paris au XIII siècle, Paris, Institut d'études augustiniennes, 1998; The Sermon, directed by B.M. Kienzle, Turnhout, Brepols, 2000.

8 Thomas de Chobham, Summa de arte praedicandi, Prologus, I (De modo significandi in theologia), cura et studio F. Morenzoni, Turnholti, Brepols, 1988, p. 10. Su quest'importante ars cfr., in particolare, F. Morenzoni, Des écoles aux paroisses cit.

9 Thomas de Chobham, Summa de arte praedicandi, VII, 2.1.2 (De inventione in narratione), ed. cit., p. 282.

10 Ars dilatandi sermones (si cita da Richard of Thetford, A Treatise on the Eight Modes of Dilatation, translated by G.J. Engelhardt, in «Allegorica», III/1 [1978], pp. 77-160, p. 104). 
nella cultura omiletica degli ordini Mendicanti e, in quanto tale, ribadito anche nell'ars di Tommaso di Chobham:

Dominus enim diversas creavit creaturas, diversas natura habentes, non solum ad sustentationem hominum, sed etiam ad doctrina eorum. Ut per ipsas creaturas non solum inspiciamus quid nobis utilis sit in corpore, sed etiam quid sit utile in anima. ${ }^{11}$

Simili indicazioni dovettero in qualche misura valicare i confini della rigida normativa delle artes per insediarsi sul diverso terreno dei predicabilia se a degno contraltare alle posizioni di Tommaso di Chobham possono essere prese alcune considerazioni premesse da Antonio da Padova alla raccolta dei suoi Sermones circa la necessità di condire con immagini e similitudini animali e naturali l'opera di edificazione spirituale promossa dal pulpito per adattarne i contenuti alle orecchie del degradato uditorio contemporaneo, ferma restante l'opportunità di far rientrare simili dilettevoli lusinghe nel rango di un ortodosso apostolato grazie all'interpretazione in chiave morale dei veicoli naturalistici:

Ad hoc nostri temporis lectorum et auditorum devenit insipida sapientia, quod, nisi verba polita, exquisita et novum quid resonantia invenerit vel audierit, legere fastidit, audire contemnit. Et ideo, ne verbum Domini, in animarum suarum periculum, eis veniret in contemptum et fastidium, in cuiuslibet evangelii principio prologum eidem consonantem, et quasdam rerum et animalium naturas et nominum etymologias, moraliter expositas, ipsi operi inseruimus. ${ }^{12}$

Di là dalle peculiarità dell' imagery zoologia antoniana,${ }^{13}$ importa qui osservare, su di un piano più generale, come, a fronte dell'alto numero di similitudini natu-

11 Thomas de Chobham, Summa de arte praedicandi, VII, 2.1.2 (De inventione in narratione), ed. cit., p. 275.

12 S. Antonii Patavini, Sermones domenicales et festivi, Prologus, curantibus B. Costa, L. Frasson, I. Luisetto, coadiuvante P. Marangon Padova, Ed. Messaggero, 1979, vol. I, p. 4. Antonio si atterrà a questa norma a tal punto che «veri e propri trattati di zoologia, di botanica e di mineralogia si potrebbero estrarre» dai suoi sermoni (F. Zambon, La simbologia animale nei "Sermones» di sant'Antonio, in Le fonti e la teologia dei "Sermoni» antoniani, Atti del Congresso Internazionale di Studio sui «Sermones» di s. Antonio di Padova, Padova, 5-10 ottobre 1981, a cura di A. Poppi, Padova, Messaggero, 1982, ora, con il titolo Sant'Antonio e gli animali, in Id., L'alfabeto simbolico degli animali cit., pp. 131-49, p. 139).

13 Sul bestiario dei sermoni antoniani cfr., almeno, F.J. Da Gama Caeiro, Nature et symbole chez s. Antoine de Padoue, in La filosofia della natura nel Medioevo, Atti del terzo congresso internazionale di filosofia medievale, Passo della Mendola (Trento), 31 agosto - 5 settembre 1964, Milano, Vita e Pensiero, 1966, pp. 363-71; B. Smalley, B. Costa, Le fonti dei «Sermones» antoniani, Padova, Messaggero, 1981, pp. 23-24 e F. Zambon, La simbologia animale nei «Sermones» di sant'Antonio cit. 
ralistiche, in particolare animalesche, nei maggiori sermonari tardo-medievali, ${ }^{14}$ l'assunto di fondo della scelta di Antonio da Padova sembrerebbe trovare concreti riscontri nella pratica apostolica di molti altri predicatori tardo-medievali, ${ }^{15}$ in questo senso agevolati non solo dall'ampia messe di notizie relative alla natura degli animali raccolta in compilazioni enciclopediche come quelle di Vincenzo di Beauvais e di Bartolomeo Anglico e dalla ricca tradizione dei bestiari propriamente detti, ma anche dalla massiccia presenza di tale materiale fantastico nel vasto numero di summae, concordanze, distinctiones e sillogi approntate per facilitare il predicatore nell'esercizio dell'apostolato, dalla fortunatissima Summa de exemplis et similitudinibus di Giovanni da san Gimignano al Liber de exemplis naturalibus di Servasanto da Faenza. ${ }^{16}$

14 Louis-Jacques Bataillon, ad esempio, rimarca l'incremento del numero di similitudini nei sermoni a partire dalla metà del XII secolo (cfr. Early Scholastic Sermons and Mendicant Preaching as Exegesis of Scripture, in Id., Ad litteram. Authoritative Texts and their Medieval Readers, Notre DameLondon, University of Notre Dame, 1992) e punta l'attenzione sull'importanza delle immagini nei sermoni tardo-medievali (Les images dans les sermones du XIII siècle, in "Freiburger Leitschrift für Philosophie und Theologie», 37 [1990], ora in Id., La prédication au XIII' siècle en France et en Italie. Etudes et documents, Torino, Variorum, 1993, pp. 327-95). Cfr. anche Id., Similitudines et exempla dans les sermons du XIII siècle, in The Bible in the Medieval World. Essay in Memory of Beryl Smalley, edited by K. Walsh and D. Wood, Oxford, Blackwell, 1985, ora in Id., La prédication au XIII siècle en France et en Italie cit., pp. 191-205 e, in un'ottica più generale, L. Bolzoni, La rete delle immagini. Predicazione in volgare dalle origini a Bernardino da Siena, Torino, Einaudi, 2002.

15 Agli studi ricordati nelle note precedenti si aggiungano C. Bremond, Le bestiaire de Jacques de Vitry († 1240), in L'animal exemplaire au Moyen Âge cit., pp. 111-21 e F. Moretti, Le rappresentazioni animali nei sermoni di Luca da Bitonto, in «Il Santo», XLIII (2003), pp. 263-93. In assenza di ulteriori studi complessivi sul bestiario dei singoli predicatori, assumono poi particolare importanza alcune osservazioni sull'argomento entro discorsi più ampi e articolati: cfr., a titolo esemplificativo, le considerazioni sul bestiario di Bernardino da Siena in L. Bolzoni, La rete delle immagini cit., pp. 220-24.

$16 \mathrm{Su}$ queste due opere, citate a titolo puramente esemplificativo, cfr., almeno, L. Oliger, Servasanto da Faenza e il suo "Liber de virtutibus et vitiis», in Miscellanea Franz Ehrke, Roma, Biblioteca Apostolica Vaticana, 1924, vol. I, pp. 148-89 e C. Delcorno, La predicazione nell'età comunale cit., p. 34, mentre a Giovanni da san Gimignano dedica alcune importanti pagine F.A. Yates, L'arte della memoria (1966), trad. it, Torino, Einaudi, 1993, pp. 78-84. Giovanni da San Gimignano, che dedica agli animali due sezioni della sua Summa (la quarta, De natabilibus et volatilibus, e la quinta, De animalibus terrestris), riprende nel prologo alcune considerazioni in materia di similituidini naturali sostanzialmente analoghe a quelle, già citate, delle artes praedicandi: «in moribus et actibus virtutum duplex habemus exemplum. [...] Hoc est igitur exemplar intrinsece cum existens in corde per fidem, scilicet Christi vita. [...] Sed est aliud exemplar extrinsecum, scilicet natura creata rerum quae sunt extra animam, quo dirigunt humana industria non tantum in operibus artium [...] sed et in moribus et actibus virtutum. [...] Nam ex ipsis naturae operibus, sumuntur similitudines et plurima exempla itilia nostris competentia moribus. [...] dum per exempla ad sensum spiritualia et subtilia declarabunt. Praedicantium igitur commodo, curioso desiderio simplicium auditorum profectui desiderans inservire, ego [...] de multarum rerum exemplis quae diversis hominum moribus et actibus adaptantur, praesens opusculum edidi» (Summa de exemplis et rerum similitudinibus locupletissima verbi Dei concionatoribus cunctisque litterarum studiosis maximo usui futura, Venetiis, 
Tuttavia, la proteiforme consistenza simbolica e allusiva del bestiario dei predicatori, pur giovandosi dei dati (pseudo-)naturalistici raccolti in simili repertori e pur trovando solido appiglio nella secolare tradizione dell'esegesi biblica, ${ }^{17}$ nella

Ex Officina Damiani Zenari, 1576, 1 recto e verso). Su simili strumenti rimane fondamentale L.J. Bataillon, Les instruments de travail des prédicateurs au XIII siècle, in Culture et travail intellectuelle dans l'Occident médiéval, Bilan des "Colloques humanisme médiéval" (1960-1980) fondés par le R.P. Hubert, publiés par G. Hasenhor e J. Longère, Paris, Editions du CNRS, 1981, pp. 197-209, mentre sulle presenze animali in simili opere si può riccorrere ad alcuni dei saggi raccolti nel già citato volume L'animal exemplaire au Moyen Âge: B. Van den Abeele, L'allégorie animale dans les encyclopédies latines du Moyen Âge, pp. 123-43; M.A. Polo de Beaulieu, Du bon usage de l'animal dans les recuils d' "exempla», pp. 147-70; F. Morenzoni, Les animaux exemplaires dans les recuilles de "Distinctiones» bibliques alphabétique du XIII siècle, pp. 171-90 e B. Bremont, L'animal comme exemple dans les encyclopédies médiévales: morale et "naturalisme» dans le "Livre des propriétés des choses", pp. 191-05.

17 Esemplare in questo senso il palinsesto di un'immagine particolarmente diffusa tra i predicatori tardo-medievali: la similitudine tra l'uomo che tura le orecchie di fronte agli insegnamenti morali dei predicatori e l'aspide che, nell'interpretazione dei bestiari medievali, per non cadere nella malia degli incantatori si chiude un orecchio con la coda e poggia l'altro al suolo per non sentire. Pur trovando un concreto parallelo nell'equivalenza nel cosiddetto Bestiario toscano tra questa natura dell'animale e coloro che "non intendeno lo bono predicatore di Dio" (Libro della natura degli animali, XVII, in Bestiari medievali, a cura di L. Morini, Torino, Einaudi, 1996, pp. 427-86, p. 445), risulta in ultima analisi tramata sulla falsariga degli empi del Salmo LVII - «Indignatio eis secundum similitudinem serpentis, sicut aspidis surdae et obturantis aures suas, quae non exaudiet vocem incantantium, et medicamenti medicati a sapiente» (5-6) - che già Agostino riferiva al comportamento di coloro che, pur non essendolo, si fanno sordi di fronte alla parola di Dio (cfr. Ennarationes in Psalmos, In Psalmum LVII, 7), contribuendo a consolidare nella tradizione esegetica un'equivalenza tra la sordità volontaria dell'aspide e coloro che ignorano il messaggio divino a causa di un eccessivo attaccamento ai beni terreni che offre ai predicatori un solido veicolo per stigmatizzare coloro che rimangono indifferenti alla morale propugnata dal pulpito. Se ne veda, a titolo puramente esemplificativo, l'impiego da parte di Bernardino da Siena in polemica contro i sodomiti: "Quinta condizione è durezza: "sicut aspidis surde": so' maliziosi. L'aspido è di sua natura sordo; e con tutto che elli abbi questo, anco ha malizia; ché non vole talvolta udire, e a questo v'aopera arte. Simile fanno questi maladetti, che perché ellino vadino a la predica, ellino non odono. "Aures habent, et non audient. Perché ellino abbino orecchie, e' non odono"; che non vogliono udire. E' li giogne la parola insino a l'orecchia, ma non passa più là» (Prediche volgari sul Campo di Siena 1427, pr. XXXIX, 85 (a cura di C. Delcorno, Milano, Rusconi, 1989, vol. II, p. 1160). Segnala la presenza della medesima immagine nei sermoni di Luca da Bitonto F. Moretti, Le rappresentazioni animali nei sermoni di Luca da Bitonto cit., p. 269, n. 16). A conferma che si tratta di un'interpretazione della natura dell'aspide, se non proprio peculiare dei predicatori, almeno ben presente all'imagery omiletica tardomedievale si veda il capitolo sull'aspide del cosiddetto bestiario di Northumberland, compilato proprio per facilitare i predicatori nella confezione dei sermoni: «Fertur autem cum ceperit pati incantatorem qui eam in quibusdam carminibus propriis evocat ut eam de caverna producat, illa cum exire noluerit, unam aurem in terram premit alteram cauda obturat et operit. Atque voces illas magicas non audiens non exit ad incantantem. Tales quidem sunt homines istius mundi qui in desideriis terrenis aurem deprimunt unam aliam vero de perpetratis ne audiant vocem Domini dicentis [....]» (IX.5.1-2 in C. White, From Ark to the Pulpit. An Edition and Translation of the "transitional" Northumberland Bestiary (13th Century), Louvain-La-Leuve, Institut d'Études Médiévales, 2009, p. 270). 
definizione della propria imagery mette a partito, accanto a dati di questa natura, anche numerose altre suggestioni legate alle tradizioni filosofica, favolistica ed esemplare del Medioevo. ${ }^{18}$

Esemplare in questo senso il palinsesto che sorregge il veicolo della similitudine con cui si apre una predica fiorentina sul digiuno di Giordano da Pisa, sulla quale si propongono qui alcune osservazioni più specifiche. Predicando dal pulpito di Santa Maria Novella durante la Quaresima del 1306 sul thema «Nunc princeps mundi huius eiecetur foras» (Io., 12, 31), ${ }^{19}$ Giordano prende le mosse dalla topica immagine delle «dure battaglie spirituali e grandi» dello spirito umano contro la «maligna cosa», intrecciando a questo sistema transuntivo quello, parimenti topico, della metafora medica per indicare la vittoria-guarigione dell'uomo sul peccato-malattia:

Sono ancora dure le battaglie spirituali e grandi, non solamente per la fatica che cci si dura, sse' combattuto da tte medesimo, ma per la durezza del nemico, ch'avemo a combattere con maligna cosa. Ma chi sse ne vuolo difendere, Cristo ne 'nsegna avere vittoria e triunfo, e inségnalci cacciare a modo ce ssi caccia la 'nfermità del corpo, e questo è in quattro modi: per dieta, per prendere medicina, $\left[\ldots . .{ }^{20}\right.$

18 Significative in questo senso le osservazioni di Giuseppe Ledda sul ruolo dell'immagine aristotelica dell'occhio del pipistrello di fronte al sole come emblema del rapporto dell'intelletto umano con le sostanze separate dalla materia nella predicazione di Giordano da Pisa e di Servasanto da Faenza: Filosofia e ottica nella predicazione medievale, in Letteratura in forma di sermone. I rapporti tra predicazione e letteratura nei secoli XIII-XVI, Atti del seminario di studi (Bologna 15-17 novembre 2001), a cura di G. Auzzas, G. Baffetti e C. Delcorno, Firenze, Olschki, 2003, pp. 53-78.

19 Già Carlo Delcorno metteva in rilievo, parlando della predicazione giordaniana, come sia «inevitabile in una predicazione ai laici [...] il "simile naturale"» e come «fra Giordano dimostri una sua tipica dignità e un'eccezionale sobrietà nell'uso di questo materiale fantastico, che penetra in tutte le manifestazioni, letterarie ed artistiche, della civiltà medievale», rilevando anche che «quando si rivolge al repertorio delle similitudini naturali, fra Giordano non dimentica il suo ideale di predicazione, diretta ad illuminare l'intelligenza di un pubblico attento e esigente» (Giordano da Pisa e l'antica predicazione in volgare cit., pp. 152-54). In generale sulla figura e sull'opera di Giordano da Pisa cfr., oltre al citato saggio di Delcorno, anche, dello stesso, La predicazione nell'età comunale, Firenze, Sansoni, 1974, pp. 38-41 e la voce Giordano da Pisa del Dizionario biografico degli italiani, vol. 55, Roma, Istituto della Enciclopedia Italiana, 2000, pp. 243-51. Cfr. anche, da ultimo, C. Iannella, Giordano da Pisa. Etica urbana e forme della società, Pisa, ETS, 1999.

20 Giordano da Pisa, Quaresimale fiorentino 1305-1306, pr. LXXIX (per cura di C. Delcorno, Firenze, Sansoni, 1975, p. 380). L'idea della pugna spiritualis, com'è noto, ha origini bibliche - cfr., ad esempio, Ef. 6, 12: "Quoniam non est nobis colluctatio adversus carnem et sanguinem, sed adversus principes et potestates, adversus mundi rectores tenebrarum harum, contra spiritualia nequitiae in caelestibus» - e ha goduto di una ininterrotta fortuna medievale, così come la serie di traslati medici per indicare la guarigione dal peccato. Sulla diffusione di quest'ultimo tipo di immagini, particolarmente presenti all'imagery dei predicatori tardo-medievali, ci si limita a rimandare a due importanti contributi attenti alle ricadute omiletiche di questo tipo di discorsi: N. Beriou, La confession dans les écrits théologiques et pastoraux du XIII': médication de l'âme ou démanche judiciaire?, in 
A questo punto, per spiegare meglio in che cosa consista il primo rimedio indicato da Cristo per vincere una simile pugna spiritualis, la "dieta», Giordano introduce, portando avanti l'imagery medica, un paragone tra l'efficacia del digiuno corporale come rimedio alle infermità del corpo e quello spirituale per vincere le infermità spirituali, prontamente specificato in riferimento a un inquietante quadretto esemplare, in cui ai demoni che vengono cacciati astenendosi dai diletti corporali viene associato il digiuno come unica risorsa per l'uomo nel cui stomaco si è introdotto un serpente per vivervi da parassita:

La dieta si è il digiuno, a modo che ssi caccia il serpente del corpo. Adiviene, quando alcun'otta altri dorme co la bocca aperta, che cci entra la serpe, e va in ventre, e llà vuole mangiare; se ttu mangi avrà che mangiare ella, nutricheralla, e ella ci starà volontieri: or già sta ella sottoterra inn-uno buco stretto, perché non potrebbe ella stare ne lo stomaco? Ma se digiuni è mistieri che ssi ne esca, quando non trova da mangiare. E però a que' cotali è dato molto consiglio pur del digiunare. A questo modo si caccia il demonio, di fare dieta e abstinenzia de le cose del mondo: se ttu le pur usi, e non ne vuoli fare alcuna dieta, in tutto questo mondo non è cosa che così notrichi il demonio come questa, non astegnendoti da le cose temporali; onde questa dieta si è uno tòrre l'arma a' demonî. ${ }^{21}$

In ultima analisi, l'intera immagine risulta tramata a partire dall'associazione tra il serpente e l'universo demonologico - «a modo che ssi caccia il serpente del corpo. [...]. A questo modo si caccia il demonio» -, diffusissima nella cultura medievale a partire da un assioma già compiutamente formulato sin dalle matrici bibliche del bestiario cristiano, nel vincolo imposto all'esegesi dall'inequivocabile accordo tra il ruolo del serpente nel dramma edenico - «Sed et serpens erat callidior cunctis animantibus terrae, quae fecerat Dominus Deus. [...]. Et ait Dominus Deus ad serpentem: Quia fecisti hoc, maledictus es inter omnia animantia et bestias terrae: super pectus tuum gradieris et terram comedes cuntis diebus vitae

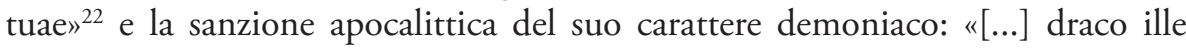
magnus, serpens aniquus, qui vocatur diabolus et Satanas, qui seducit universum orbem $[\ldots] » .{ }^{23}$ Alle radici bibliche della demonologia medievale, però, è possibile

L'aveu. Antiquité et Moyen Âge, Actes de la table ronde organisée par l'École française de Rome avec la concours de CNRS et de l'Université de Trieste, Rome 28-30 mars 1984, Palais Farnese, École française de Rome, 1986, pp. 261-82 e C. Iannella, Malattia e salute nella predicazione di Giordano da Pisa, in «Rivista di storia e letteratura religiosa», XXX (1995), 2, pp. 177-216.

21 Giordano da Pisa, Quaresimale fiorentino 1305-1306, pr. LXXIX (ed. cit., p. 380).

22 Gn., 3, 1 e 14.

23 Apoc. 12, 9. Si tratta di un assunto ripetuto, praticamente alla lettera, a Apoc. 20, 2: «Et aspprendit draconem, serpentem antiquum, qui est diabolus et Satanas, et ligavit eum per annos mille, et misit eum in abyssum [...]». Per una prima informazione circa il valore del serpente nella 
ricondurre non solamente l'equivalenza di fondo della similitudine in esame, ma anche alcuni aspetti più specifici del comportamento del serpente di Giordano. In particolare, il suo albergare, in qualità di parassita, nelle viscere dell'uomo sembra riflettere, variandola sensibilmente, l'idea, parimenti topica nell'imagery medievale, secondo cui il diavolo si insinua nel corpo degli uomini per farvi proliferare dall'interno pericolose inclinazioni peccaminose, destinata a una lunga fortuna a partire da alcune declinazioni evangeliche della demonologia biblica, all'incrocio tra il fugace accenno alla guarigione di Maria di Magdala - «de qua septem daemonia exierant» $-{ }^{24}$ e il motivo degli spiriti immondi che fissano la loro dimora nell'uomo:

Cum autem immundus spiritus exierit ad homine, ambulat per loca arida quaerens requiem et non invenit. Tunc dicit: Revertar in domum meam, unde exivi. Et veniens invenit eam vacantem, scopis mundatam et ornatam. Tunc vadit et adsumit septem alios spiritus secum nequiores se, et intrantes habitant ibi: et fiunt novissima hominis illius peiora prioribus. Sic erit et generationi huic pessimae. ${ }^{25}$

Un'idea, quest'ultima, che già prima di Giordano sembra intrecciarsi con alcune proprietà specifiche tradizionalmente associate al serpente, quali la tortuositas e la lubricitas, a coniare per l'animale l'immagine di un essere insinuante, effige del demonio anche in quanto capace di strisciare sin nel cuore degli uomini corrompendolo dal di dentro:

Diabolus enim est serpens lubricus, cuius si capiti, id est primae suggestioni, non resistitur, totum in interna cordis, dum non senti-

Bibbia e nell'esegesi cristiana antica cfr. M.P. Ciccarese, Serpente cit., a cui si rimanda anche per una rassegna di luoghi paralleli a quelli indicati, a titolo puramente esemplificativo, a testo. Cfr. anche H. Egli, Il simbolo del serpente (1982), trad. it., Genova, ECIG, 1993 e F. Giunta, Serpenti, in Animali della letteratura italiana, a cura di G.M. Anselmi e G. Ruozzi, Roma, Carocci, 2010², pp. 246-53.

24 Lc., 8, 2: «et mulieres aliquae, quae erant curatae a spiritibus malignis et infirmitatibus, Maria, quae vocatur Magdalene, de qua septem daemonia exierant [...]». Tale accenno biblico viene poi espanso in riferimento alle proprietà specifiche di ciascun demone nella tradizione esegetica. Cfr., ad esempio, il commento ad loc. di Hugues de Saint-Cher: «Sacri expositores volunt ut per septem demonia intelligantur universa peccata, quia septenarius numerus est universitatis. Dicamus ergo daemonia septem, scilicet, universitas peccatorum, seu septem criminalia, quae septem nominibus daemonum repertis in sacra scriptura conveniunt, quae sunt Diabolus, Behemoth, Leviathan, Asmodeus, Satanas, Exterminator, Daemon» (Hugonis Cardinalis Opera Omnia in Universum Vetus et Novum Testamentum, Venetis, apud Nicolaum Pezzana, 1706, vol. VI, p. 176).

$25 \mathrm{Mt} .12,43-45$. Di qui prende le mosse il motivo del demone che si introduce nell'animo dell'uomo, che assume il peso e la consistenza di un vero e proprio tópos in alcune declinazioni della letteratura tardo-antica e medievale. Cfr., ad esempio, P.F. Beatrice, L'allegoria nella "Psychomachia» di Prudenzio, in «Studia Patavina», 17 (1971), pp. 25-73. 
tur, illabitur. Tunc contra eum quem possidet diabolus acrius saevit, quando se virtute divina ad eo expellendum agnoscit. ${ }^{26}$

Si tratta, però, non solo di dati culturali di generale divulgazione, inadatti quindi a stringere legami vincolanti con l'immagine giordaniana, ma anche di tópoi capaci di dar ragione solamente delle sovrastrutture simboliche più generali alluse nella similitudine del predicatore toscano, ma non degli aspetti più peculiari dell'attiva rielaborazione a cui Giordano ha sottoposto la solida trafila di immagini topiche del serpente e, quindi, dei tratti più specifici dell'exemplum addotto nella predica fiorentina. Occorre, infatti, tenere presente che il motivo della serpe in corpo, pur presente in nuce in alcune delle evoluzioni medievali del motivo evangelico della possessione diabolica, non sembra però tradursi in formulazioni in grado di dar pienamente contro del passo giordaniano in questione. Sono, nello specifico, quei dati che assicurano maggiore concretezza esemplare all'immagine simbolica del serpente a segnare lo iato tra simili impalcature simboliche e la specifica rilettura giordaniana del motivo del serpente-diavolo che si insinua nel corpo di un uomo: da un lato, l'accurata descrizione delle modalità con cui l'animale penetra nell'organismo, e, dall'altro, la precisa individuazione nel digiuno dell'unico rimedio efficace per guarire da un simile morbo. Sono elementi che non trovano immediato riscontro né nelle fila della suprema opera di sintesi dei dati biblici circa il comportamento dei serpenti e delle lezioni di erpetologia dei naturalisti classici promossa nelle enciclopedie tardo-medievali, ${ }^{27}$

26 Defensor Locociagensis, Scintillarum liber, LXXVII, in Patrologia latina cursus completus series latina, accurante J.P. Migne, Paris, Garnier, 1844 sgg., vol. LXXX, col. 711.

27 Cfr., a titolo puramente esemplificativo, il consuntivo della natura dei serpenti offerto da Tommaso di Cantimpré in apertura della sezione dedicata ai serpenti del Liber de natura rerum: «Generaliter primo dicendum est de serpentibus. Aristoteles: Nullus serpens habet testiculos, sed via sicut picis, et matrix eius est longa. In aliis interioribus est sicut lacertulus. Lingua serpentis est subtilis, longa, simile reni. [...]. Terra serpentem homine percusso, ut dicit Plinius, nichil aliud est quam fel serpentium, et inde ad venas sub spina et postea ad os pervenit vel ad caudam ut in scorpionibus. [...]. Iacobus et Ysidorus: Sunt itaque serpentes natura fervidi, unde nil vel parum nocent, postquam infriguerunt. Nocte autem minus ledunt quam die, eo quod nocturno rore frigescunt. Hyemali frigore in nodos torquentur, estate vero resolvuntur. [...] Refert Ambrosius quod ieiuni hominis sputum serpentem necat. Nam si serpens ex eo vel parum contigerit, olico moritur. [...]. Ut dicit Experimentator: tot sunt venena, quot serpentium genera, tot pernices quot species, toto dolores quot colores habent. [...]. Vestigia serpentium, ut dicit Ysidorus, talia sunt, ut pedibus carere videantur, cortis tamen et squamarum nisibus repunt, quas a summo gutture usque ad ima parili ordine dispositas habent; squamis quasi unguibus. Caput abscondens serpens totum corpus obiecit ferienti; nam si caput ipsius evaserit, nichilhominus vivit amisso corpore. Ingressus aqua venenum deponit; postquam autem aquas exierit, venena resumit. [...]. De medullis hominum serpentes procreari dicuntur, et hoc maxime in spina dorsi» (VIII, 1, editio princeps secundum codices manuscriptos, Teil I: Text, Berlin-New-York, Walter de Gruyter, 1973, pp. 276-77). Si noti, almeno di sfuggita, come la credenza secondo cui il serpente «per foramen petre transit» (ivi, p. 277), diffusissima sin dal Physiologus nella tradizione enciclopedica e nei bestiari medievali, sembra riflettersi 
né nella tradizione dei bestiari propriamente detti, impegnati a rielaborare simbolicamente quelle medesime proprietates sia per argomentare l'apparentemente paradossale possibilità di una interpretazione in bonam partem della natura del serpente sia per arricchire con ulteriori riferimenti a comportamenti specifici la tradizionale interpretazione in chiave negativa e demonologica del rettile. ${ }^{28}$

Piuttosto che nella tradizione dei bestiari e delle enciclopedie naturalistiche stricto sensu, il primo degli aspetti apparentemente stravaganti del serpente di Giordano da Pisa, il suo insinuarsi nella bocca di un uomo che dorme per insediarsi nel suo stomaco, sembra, invece, trovare singolare riscontro in alcune declinazioni cristiane del più generale motivo folklorico della presenza di un animale vivo nel corpo di un uomo. Tra le infinite variazioni di questo tópos, infatti, è documentato anche il caso in cui il doloroso parassita è proprio un serpente: in alcune versioni del Panchatantra, ad esempio, si legge di come un principe "wasted daily in every limb because of a snake that used his belly as a home instead of an ant-hill» e di come questi riesca a guarire solo quando la moglie viene a conoscenza del rimedio necessario a uccidere l'animale ascoltando un litigio tra il serpente che vive nello stomaco del marito, momentaneamente uscito dalla bocca dell'uomo per prendere aria, e un altro serpente, che grida contro al primo proprio il nome dell'unico alimento in grado di uccidere il parassita. ${ }^{29}$ Pur nell'incolmabile distanza tra un simile racconto e gli esisti della

anche nella domanda retorica impiegata da Giordano da Pisa per convincere il suo uditorio che il serpente può effettivamente vivere nello stomaco di un uomo: «[...] or già sta ella sotterra inn uno buco stretto, perché non potrebbe ella stare ne lo stomaco?».

$28 \mathrm{Si}$ veda, di nuovo a titolo puramente esemplificativo, il capitolo «De la natura del serpente» del Libro della natura degli animali, bestiario tardo-duecentesco prodotto in Italia del Nord: «Serpente quando è vecchio si rinovella in questo modo che udite: che elli digiuna tanto che tucta la sua pelle trema e nulla rimane se non tanto l'ossi e la carne; et quando è cusì ordinato, cerca uno strecto partuso di pietra che appena vi passi, e passa per lo mezo del partuso, e cusì passando la sua pelle vecchia da la carne si parte. [...]. Questo che fa lo serpente vecchio, di rinnovarsi digiunando molto et che entra per lo partuso de la pietra e quine lassa la pelle vecchia, e cusì aviene dell'omo peccatore, che tanto stea indei peccati di fina ch'elli è vecchio; al quale è bizogno che digiunando e affrigendo lo su corpo e contringendo, et Dio con buone orasione pregando, ch'elli abbia misericordia di lui, e cusì purga de li suoi peccati ed è facto nuovo; e cusì è quando elli esce dal battismo e trova vita eterna» (XLV, ed. cit., pp. 464-65).

29 Si cita dalla traduzione inglese del Panchatantra di Arthur W. Ryder (Chicago, University of Chicago, 1925, pp. 346-48), in considerazione del fatto che il racconto, presente solo in alcune versioni della raccolta, è stato escluso nella ricostruzione testuale condotta da Francklin Edgerton: Panchatantra Reconstructed, New Haven, American Oriental Society, 1924. Per le versioni della raccolta che contengono il racconto citato si veda l'utile tabella riassuntiva in appendice a The Pañcatantra. The Book of India folk Wisdom, a new translation by P. Olivelle, Oxford, Oxford Univeristy Press, 1997, pp. 162-64. Per la diffusione e la consistenza di tale motivo folklorico si vedano i materiali raccolti in Motif-index of folk-literature: a classification of narrative elements in folktales, ballads, myths, fables, mediaeval romances, exempla, fabliaux, jest-books and local legends, revised and enlarged edition by S. Thompson. Bloomington-London, Indiana University Press 1966, in parti- 
similitudine giordaniana, risulta però evidente la sostanziale affinità di fondo tra il ruolo del serpente nella predica fiorentina e lo schema essenziale di un simile motivo folklorico, entrambi incentrati sui problemi causati dalla presenza di un serpente nel ventre di un uomo.

Tuttavia, di là dalla possibile circolazione europea di questo genere di racconti, ai fini del presente discorso preme piuttosto rilevare come le suggestioni qui offerte circa l'atteggiamento del serpente nei confronti dell'uomo, in particolare l'eventualità che questi possa albergare nelle sue viscere, pur non trovando accoglienza tra le nature comunemente chiamate in causa per l'interpretazione simbolica dell'animale nei bestiari e nelle compilazioni enciclopediche, godettero, però, di una particolare fortuna nell'ambito della letteratura agiografica e miracolistica mediolatina. Gregorio di Tours, ad esempio, annovera tra i miracoli di santa Monegonda la guarigione di un fanciullo il cui corpo soffre dei morsi di serpenti velenosi generatisi al suo interno a seguito dell'assunzione di una particolare bevanda finché la santa non lo libera imprimendogli sul ventre una foglia di vite sporca della sua saliva e segnata con la croce:

Puer vero incola loci maleficium in potione hausit, de quo medificatus, ut asserunt, serpentes generati in intraneis pueri magnum dolorem suis morsibus excitabant, ita ut nulli quiescendi mora vel modicum momentum indulgere. Sed neque cibum aut potum capere poterat, et si post diu aliquid accipiebat, protinus reijciebat. Qui adductus ad beata feminam, petiit se ejus virtute mundari. Cumque illa reclamaret indigna se esse quae haec agere posset, implicita peccatu parentum, ventrem pueri palpat, et palma demulcet; sensit ibi anguium venenatorum nequitia latitare. Tunc acceptum pampini viridis folio, saliva linivit, fixitque super eum crucis beatae signaculum, quod ponens super alvum juvenculi, dolore paulum sedato, obdormivit in scamno, qui olim doloribus insistentibus caruerat somno. Post unius vero horae momentum consurgens, ad purgandum ventrem egressus, pestiferae generationis germen effudit, gratiasque referens ancilla Dei, sanus abscessit. ${ }^{30}$

In questo caso, pur nella sostanziale affinità di fondo, le differenze rispetto alla similitudine di Giordano da Pisa sono però notevoli, interessando alcuni snodi fondamentali della vicenda: dal fatto che in Giordano il serpente entra nella bocca di un dormiente mentre qui i serpenti si generano direttamente nell'intestino del malcapitato in seguito all'ingestione di un cibo particolare, all'evidente diversità

colare per il serpente si vedano le indicazioni rubricate alla voce G.328.1.

30 Vitae patrum, XIX, in Patrologia latina cit., vol. LXXI, col. 1090. Gregorio riferisce il medesimo miracolo anche nel XXIV capitolo del De gloria beatorum confessorum. In generale sul ruolo degli animali nella tradizione agiografica cfr. P. Boglioni, Les animaux dans l'hagiographie monastique, in L'animal exemplaire cit., pp. 51-80. 
dei rimedi grazie ai quali la vicenda volge a lieta conclusione. Di là da queste, pur notevoli, differenze, importa però osservare come l'appropriazione del motivo della serpe in corpo nel contesto, occidentale e cristiano, del racconto di un miracolo compiuto da una santa determini l'intrusione nello schema del tópos della serpe in corpo di quella medesima connotazione demoniaca del serpente su cui farà perno l'exemplum di Giordano da Pisa, assente invece negli archetipi orientali del motivo. Tuttavia, se nel caso del miracolo di santa Monegonda una simile associazione, per quanto desumibile dal contesto del racconto, non viene pienamente esplicitata, in alcune ulteriori riscritture agiografiche del medesimo motivo una simile identificazione è proposta in termini più espliciti. È il caso, ad esempio, di un miracolo attribuito a Pietro il Venerabile. A quest'ultimo viene sottoposto il caso, apparentemente disperato, di un uomo il cui corpo è infestato da un serpente, che Pietro riesce a liberare estraendo il rettile dalla bocca dell'uomo durante una messa. Qui, l'associazione tra il serpente e la sfera del demoniaco diviene perfettamente evidente quando si precisa che Pietro ha estratto il parassita dal corpo dell'uomo proprio quando, nella messa, si declamava l'investitura evangelica indirizzata da Cristo agli apostoli, in cui il nesso tra il rettile e i demoni risuona distintamente nel parallelismo a distanza della duplice definizione dell'azione salvifica della missio apostolorum ("[...] in nomine meo daemonia eicient, linguis loquentur novis, serpentes tollent, $[\ldots] »):^{31}$

Tempre vero quo vir beatus ab Hispaniis remeabat [...] venit ad eum quidam monachus, nomine Jordanus, [...], consilium querens super morbo quod acciderat rustico suo. Dicebat enim rusticus serpentem corpis suum intrasse, et hac occasione se assidue torqueri et per singula momenta proximum mortu fieri. [...]. Celebravit autem missam de Ascensione, et illum qui torquebatur fecit adesse. Cum vero Evangelium legeretur, et ad illum locum venietur, ubi dicitur: Serpentes tollent, miser os aperuit, et serpens caput emisit, quem pater manu accipiens, et a corpore hominis exthraens, cunctis qui aderant stupentibus, in medium projecit. ${ }^{32}$

L'interesse del racconto del miracolo di Pietro ai fini del presente discorso, però, non si limita all'esplicitazione del nesso tra la serpe e l'universo della negatività demoniaca, ma interessa anche alcuni altri particolari del quadro esemplare della predica di Giordano. In questa versione del miracolo, infatti, la serpe non si trova da sempre nel corpo dell'uomo - come nel racconto indiano del Panchatantra -, né vi si ingenera a seguito dell'ingestione di un particolare cibo o di una particolare bevanda - come nel caso del fanciullo guarito da santa Monegonda -, ma si insedia

31 Cfr. Mc. 16, 17-18.

32 Rodulpho Monacho, Vita Petri Venerabilis, 16, in Patrologia latina cit., vol. CCLXXXIX, col. 27. 
nel l'organismo penetrandovi da qualche pertugio, come sembra lasciar intendere l'impiego del verbo intrare nell' icastico resoconto del malanno che affligge il «rusticus» («Dicebat enim rusticus serpentem corpis suum intrasse»), esattamente come, nella similitudine di Giordano, il serpente "cci entra [...] e va in ventre».

A fronte di questa notevole affinità, però, occorre precisare che, mentre nel racconto del miracolo attribuito a Pietro il Venerabile risulta del tutto assente qualsiasi riferimento alle modalità con cui il rettile si sia introdotto nel corpo dell'uomo, a quest'aspetto del racconto esemplare presterà invece particolare attenzione Giordano, sottolineando come la serpe raggiunga il ventre introducendovisi dalla bocca dell'uomo: «quando alcun'otta altri dorme co la bocca aperta, $[\ldots]$ cci entra il serpente, e va in ventre». Soccorrono in questo senso alcune ulteriori versioni del motivo della serpe in corpo, in cui gli snodi narrativi della vicenda assumono contorni ancora più prossimi a quella che sarà la riscirttura giordaniana del motivo. Vincenzo di Beauvais e Iacopo da Varazze, ad esempio, concordano nel riferire, tra i miracoli post mortem dei santi Cosma e Damiano, anche quello della liberazione del corpo di un uomo - di nuovo un «rusticus» - da una serpe:

Rusticus quidam cum post laborem messis in campo aperto ore dormiret, serpent in eius ventrem ingressus est. Evigilans autem cum nihil sentiret domum rediit et sero facto gravissime torqueri se sensit. Voces autem miserabiles emittebat et sanctos dei Cosmam et Damianum in sui auxilium invocabat, sed cum dolor semper incresceret ad ecclesiam sanctorum martyrum confugit et ibidem eo subito dormiente serpens sicut intraverat per eius exiuit. ${ }^{33}$

In questa versione del miracolo, infatti, l'attenzione per le modalità di ingresso della serpe nel corpo dell'uomo, oltre a documentare una sensibilità per determinati aspetti della vicenda a cui non si rivelerà insensibile neppure Giordano, contribuisce ad arricchire la scenetta esemplare di alcuni particolari che trovano riscontro nell'exemplum giordaniano: anche qui, infatti, il serpente entra nel corpo dell'uomo approfittando del fatto che, addormentandosi con la bocca aperta, il «rusticus» ha offerto al rettile una via d'ingresso privilegiata al proprio corpo («cum post laborem messis in campo aperto ore dormiret, serpent in eius ventrem ingressus est»). Il tema della bocca come possibile accesso per il serpente agli intestini dell'uomo, però, non si trova solamente nella tradizione agiografica relativa ai santi Cosma e Damiano, ma anche in alcune altre riscritture del medesimo tópos. Particolarmente vicino alla similitudine giordaniana sembra in questo senso essere, ad esempio, uno dei miracoli rubricati nel decimo libro del Dialogus

33 Iacopo da Varazze, Legenda aurea, CXXXIX (edizione critica a cura di G.P. Maggioni, Sismel, Edizioni del Galluzzo, 1998, vol. II, p. 979). Cfr. anche Vincenzo di Beauvais, Speculum historiale, XXII, 44. 
miraculorum di Cesario di Heisenbach, dove si racconta di come un serpente sia entrato nel ventre di una donna incinta passando attraverso la bocca, che questa teneva aperta mentre dormiva nei pressi di un alveare:

Simile pene contigit in Diocesi Traiectensi in villa Dulre. Nutrix Allardi novicii nostri, sicut ipse mihi retulit, cum quadam die iuxta alveola apum dormiret, ingens serpens per os ventrem illius intravit. Adveniente eius marito ut dormientem excitaret, vidit caudam ad mensuram digiti eiusdem serpentis adhuc superesse. Quem cum retrahere non posset, illa evigilavit dicens: "Valde incommode soporata sum". Et noluit ei maritus indicare quod acciderat, ne ex ipso timore moreretur. Erat enim vicina partui. Cui a matre consilio viri lac et dulcia quaeque ministrabantur, ut serpens eisdem potatus, quiesceret.

In hora vero partus mulier cum infante serpentem effudit. Quem cum vir extracto gladio exspectasset, et ille sicut est naturaliter prudens, ad feminam rediret, praeoccupatis eius itineribus occisus est. Adhuc eadem mulier vivit. ${ }^{34}$

Tuttavia la versione del motivo offerta da Cesario di Heisenbach non si limita a riprendere questo particolare, ma sembra introdurre nel racconto della vicenda miracolosa anche alcuni altri aspetti che tornano nella predica di Giordano da Pisa. Uno dei maggiori punti di scarto del miracolo registrato da Cesario rispetto alle altre attestazioni del tópos della serpe in corpo consiste, infatti, nell'accenno al fatto che il marito, per mantenere calmo l'animale che alberga nelle viscere della moglie, le somministra particolari tipi di cibo nella speranza che anche il serpente se ne possa nutrire e, quindi, placarsi. ${ }^{35} \mathrm{Si}$ tratta di un particolare che, secondario nell'economia del racconto di Cesario, assume pieno valore qualora sia considerato in relazione all'insistenza di Giordano da Pisa sul fatto che la serpe che vive nello stomaco dell'uomo si nutre del suo stesso cibo: «[...] cci entra la serpe, e va in ventre, e llà vuole magiare; se ttu mangi avrà che mangiare ella, nutricheralla, e ella ci starà volontieri». Pur nelle evidenti affinità in questo senso tra le versioni offerte da Cesario e da Giordano del tópos della serpe in corpo, occorre però precisare che quello che nel Dialogus rimane un aspetto secondario

34 Dialogus miraculorum, X, 72 (eingeleitet von H. Schneider, übersetzt und kommentiert von N. Nöges und H. Schneider, Turnhout, Brepols, 2009, vol. IV, pp. 2030-32). Si osservi, seppure di sfuggita, come nelle note al testo i curatori del volume non manchino di segnalare che "Das ein Tier, insbesondere eine Schlange, in einen Menchen eindringt und wieder aus ihm lebend hervorkommt, ist ein verbreitetes Motiv des Erzählliteratur» (ed. cit., p. 2030, nota 1943).

35 Anche le convinzioni circa la predilezione da parte dei serpenti di determinati tipi di cibi sono un elemento presente tra le proprietates tradizionalmente riferite all'animale nelle compilazioni enciclopediche. Cfr., ad esempio, Tommaso di Cantimpré, Liber de natura rerum, VII,1: «Serpens multum desiderat vinum; unde vino serpentes solent allicere qui eos venantur [...]» (ed. cit. p. 276). 
e marginale rispetto al filo principale della narrazione miracolosa, nella predica giordaniana diviene l'asse portante dell'intera scenetta esemplare. È proprio in virtù del fatto che il serpente, una volta entrato nell'organismo, si nutre del medesimo cibo ingerito dall'uomo nel cui stomaco si è insediato che il predicatore toscano può assumere il tópos della serpe in corpo a degno exemplum delle virtù del digiuno: come il digiuno, deludendone le aspettative, induce il serpente corporale ad abbandonare il corpo nel quale si era insediato, così il serpente spirituale, la demoniaca inclinazione peccaminosa che si impossessa dell'uomo, viene scacciato dalla rinuncia alle lusinghe dei beni terreni, ossia dal digiuno spirituale del buon cristiano. «Ma se digiuni è mistieri che ssi ne esca, quando non trova da mangiare. [...]. A questo modo si caccia il demonio, di fare dieta e abstinenzia de le cose del mondo" chiosa, infatti, Giordano da Pisa. Il differente peso accordato da Cesario e Giordano alla natura parassitaria del serpente che alberga nel ventre umano, così come l'assenza di riferimenti a quest'aspetto della vicenda negli altri testi passati in rassegna, sembrerebbe, d'altronde, trovare una prima spiegazione nel fatto che, per Giordano, quest' elemento è funzionale soprattutto per additare nel digiuno il rimedio più efficace in simili evenienze, volgendo così il discorso all'interpretazione morale del veicolo animalesco: un dato, quest'ultimo, che non può trovare ragione in racconti, come le testimonianze agiografiche sopra ricordate, in cui è giocoforza che la soluzione della vicenda sia affidata alla vis miracolosa del santo e non alla pratica quotidiana di un'etica cristiana tutta terrena.

L'aspetto forse più innovativo della similitudine di Giordano rispetto agli analoghi racconti agiografici sembra, infatti, risiedere proprio nel legame istituito tra il motivo della serpe in corpo e il tema del digiuno e, nello specifico, nell'individuazione dell'astensione dai cibi come unico modo per scacciare l'insidioso parassita dal proprio corpo. Del tutto assente nella teoria di rimedi proposti nei testi sinora considerati, quest'aspetto della similitudine giordaniana sembra, però, intrattenere precisi rapporti con una particolare natura del serpente tra quelle passate in rassegna nelle compilazioni enciclopediche tardo-medievali. A partire, almeno, da un passo dell'Hexameron di Ambrogio, ${ }^{36}$ infatti, prende le mosse la convinzione che il serpente muoia qualora entri in contatto con la saliva di un uomo a digiuno, prontamente interpretata come argomento sufficiente a dimostrare la poderosa forza di questa pratica ascetica nell'abbattere tanto le insidie corporali quanto quelle spirituali. "Vides, o homo, quanta vis ieiuni sit, ut et sputo suo homo terrenum serpentem inficiat et merito spiritualem», ${ }^{37}$ ammonis-

36 Cfr. Hexameron, VI, 28: "Jejuni hominis sputum si serpens gustaverit, moritur. Vides quanta vis jejunii sit; ut et sputo suo homo terrenum serpentem interficiat, et merito spiritalem».

37 Tommaso di Cantimpré, Liber de natura rerum, VIII, 1: «Refert Ambrosius quod ieinui hominis sputum serpentem necat. Nam si serpens ex eo vel parum contigerit, ilico moritur. Vides, o homo, quanta vis ieiuni sit, ut et sputo suo homo terrenum serpentem inficiat et merito spiritualem» (ed. cit., p. 277). 
ce, infatti, Tommaso di Cantimpré dopo aver riferito di questo tallone d'Achille del serpente sulla base di un trasferimento di senso dalla battaglia corporale con il rettile a quella spirituale contro le forze del male a cui ricorre anche Antonio da Padova in uno dei suoi sermoni, a conclusione di una catena di concordanze bibliche sull'efficacia del digiuno: «Saliva hominis ieiuni occidit serpentem. Magna virtus ieiunii, quae pestes animae curat, antiqui hostis fraudem exsuperat». ${ }^{38}$

L'originalità della versione giordaniana del motivo della serpe in corpo sembrerebbe, in questo senso, definirsi all'incrocio tra la ripresa di un quadretto esemplare diffuso nella tradizione agiografica e miracolistica medievale - i disagi causati dalla presenza di un serpente nel corpo di una persona - e la fiducia accordata alla leggenda, ben presente agli enciclopedisti tardo-medievali, secondo cui il serpente possa essere ucciso da un uomo che osserva rigorosamente il digiuno. A conferma di una simile ipotesi potrebbero essere prese le parole con cui Vincenzo di Beauvais argomenta, nello Speculum morale, la capacità del digiuno di sconfiggere le tentazioni diaboliche, in cui, fermo restante il riferimento alla topica equivalenza serpente-diavolo, all'elogio della virtus benefica della saliva dell'uomo si sostituisce la ripresa di un sistema transuntivo particolarmente prossimo a quello impiegato da Giordano nella similitudine in esame, quello dell'estrazione del peccato:

Item discretum ienunium ipsum diabolum fugat. [...]. Sicut animalia venenosa a se expellunt acriora medicamenta, ita ieunium discreutum cogitationes diabolicas, immo etiam ipsum diabolum. Vulgarites dicitur fames expellunt lupum a nemore; et ienium dicretum diabolum a umbroso corde. [...]. sic diabolus in sicco ieniunio vincitur. [...]. Item ieunium vincit hostes, serpentes, et dracones corporales, et etiam spirituales. ${ }^{39}$

"Sicut animalia venenosa a se expellunt acriora medicamenta, ita ieunium discreutum cogitationes diabolicas, immo etiam ipsum diabolum»: è questo, d'altronde, l'assunto teologico che Giordano si propone di meglio illustrare con l'exemplum della serpe in corpo, che nell'economia della predica fiorentina serve proprio a dimostrare che «a modo che ssi caccia il serpente del corpo. [...]. A questo modo si caccia il demonio, di fare dieta e abstinenzia de le cose del mondo».

38 S. Antonii Patavini, Sermones domenicales et festivi, Dominica I post octavam epiphanie: "Item, ieiunium purificat, unde in Ioele: "Convertimini ad me in toto corde vestro, in ieiunio et fletu et planctu "; et in Matthaeo: "Tu autem cum ieiunas, unge caput tuum et faciem tuam lava ". Moyses post ieiunium quadraginta dierum legem Domini immaculatam, convertentem et purificantem animas, meruit accipere, et Elias sibilum aurae tenuis audire. Saliva hominis ieiuni occidit serpentem. Magna virtus ieiunii, quae pestes animae curat, antiqui hostis fraudem exsuperat».

39 Speculum morale, III, x, 27 (Duaci, ex Officina typographica Baltazaris Belleri, 1624, rist. anast. Graz, Akademische Druck Verlagsanstalt, 1964, col, 1504-07). 